\title{
ON THE CONVERGENCE AND OVERCONVERGENCE OF SEQUENCES OF POLYNOMIALS OF BEST SIMUL- TANEOUS APPROXIMATION TO SEVERAL FUNCTIONS ANALYTIC IN DISTINCT REGIONS*
}

BX

\section{J. L. WALSH AND HELEN G. RUSSELL}

1. Introduction. The purpose of this paper is to present some theorems on the convergence and overconvergence of sequences of polynomials of best approximation to a function $f(z)$ analytic on a closed limited point set whose complement is of multiple (finite or infinite) connectivity. Our main theorem is the following:

THEOREM I. Let $M$ be an arbitrary closed limited point set of the z-plane whose complement $K$ is connected and possesses a Green's function with pole at infinity. $\dagger$ Let $w=\omega(z)$ be a function which maps $K$ (conformally but not necessarily uniformly) onto the exterior of the unit circle in the w-plane so that the points at infinity in the two planes correspond to each other. Let $C_{R}$ denote the transform (i.e., in $K$ ) of $|w|=R, R>1$, under the mapping function $w=\omega(z)$.

(1) If the function $f(z)$ is analytic and single-valued on and within $C_{R}$, there exist polynomials $P_{n}(z)$ of respective degrees $n \ddagger, n=1,2, \cdots$, such that the inequalities

$$
\left|f(z)-P_{n}(z)\right| \leqq N / R^{n}, z \text { on } M, R>1,
$$

where $N$ is dependent on $R$ but not on $n$ or $z$, are valid for every $z$ on $M$.

(2) If there exist polynomials $P_{n}(z)$ of degree $n, n=1,2, \cdots$, such that (a) is valid for every $z$ on $M$, then the sequence $\left\{P_{n}(z)\right\}$ converges interior to $C_{R}$, uniformly on any closed point set interior to $C_{R}$, and thus $f(z)$ is analytic $\$$ throughout the interior of $C_{R}$.

* Presented to the Society, October 29, 1932; received by the editors February 27, 1933.

$\dagger$ The requirement that $K$ should possess a Green's function is equivalent to the requirement that $K$ should be regular, in the sense that the Dirichlet problem (for arbitrary continuous boundary values) can be solved for $K$. See Kellogg, Proceedings of the National Academy of Sciences, vol. 12 (1926), pp. 397-406.

I A polynomial of degree $n$ in $z$ is any expression of the form $a_{0}+a_{1} z+a_{2} z^{2}+\cdots+a_{n} z^{n}$.

$\S$ If $f(z)$ is not originally assumed to be defined on the entire point set considered, then the definition in the new points is to be made by analytic extension interior to $C_{R}$, or, what amounts to the same thing, by means of the convergent sequence of polynomials. 
The Green's function $G(x, y)$ with pole at infinity for the region $K$ is (1) harmonic in $K$ except at infinity where $G(x, y)=\log r+G_{1}(x, y)$, $r=\left(x^{2}+y^{2}\right)^{1 / 2}$, and $G_{1}(x, y)$ is harmonic at infinity, and (2) $G(x, y)$ is continuous and vanishes on the boundary of $K$.

It will be noticed that the hypothesis on the point set $M$ is satisfied provided $M$ is closed, limited, without isolated points, and provided $K$ is connected and of finite connectivity.

This theorem is known for the case that the complement of $M$ is simply connected and that $M$ is not a single point. The second part of the theorem for that case is due to Walsh and the formulation of the entire theorem together with detailed references was published by him.* Among the writers to whom various parts of the theorem are due are Faber, S. Bernstein, M. Riesz, Fejér, and Szegö; the theorem for the case that $M$ is a segment of the axis of reals is due to Bernstein. The generalization to be proved here is made possible by the consideration of the equipotential curves for the infinite region $K$ and of approximation by them to the boundary of $K$, by the approximation to analytic curves by lemniscates, and finally by the use of a sequence of polynomials found by interpolation.

By means of Theorem I we shall derive some results on convergence and overconvergence,--results which are generalizations of results already established by Walsh $\dagger$ in the less general case mentioned. We study also the convergence of sequences of polynomials of best approximation, where best approximation is measured (1) in the sense of Tchebycheff, (2) by line integrals taken over rectifiable Jordan curves bounding the point set considered, (3) by surface integrals taken over the region considered. The two latter methods of approximation yield interesting results in regard to polynomials belonging to a point set, a problem which has been considered in the case of a simply connected region by Faber, Fejér, Szegö, Bergmann, Bochner, Carleman, and Smirnoff. $\ddagger$ All three methods of approximation yield results on the exact region of uniform convergence of the sequence of polynomials of best approximation and show that this region depends not merely on the singularities of the given function $f(z)$ but also on the monogenic character of the function $f(z)$.

The term overconvergence is used in the sense of Walsh to denote that if a sequence of polynomials converges sufficiently rapidly on a point set $M$ of the kind described, then that sequence necessarily converges also on a cer-

* Münchner Berichte, 1926, pp. 223-229.

† These Transactions, vol. 32 (1930), pp. 794-816; these Transactions, vol. 33 (1931), pp. 370388. We shall refer to these papers as (1) and (2) respectively.

$\ddagger$ Detailed references are given below. 
tain larger point set containing $M$ in its interior.

2. Approximation by analytic curves to the boundary of a given point set. We shall prove several lemmas.

Lemma I. Let $M$ be a closed limited point set of the z-plane whose complement $K$ is connected and possesses a Green's function $G(x, y)$ with pole at infinity. Then $w=\omega(z)=e^{G+i H}$, where $H$ is conjugate to $G$, is a function which maps $K$ onto the exterior of the unit circle in the w-plane so that the points at infinity in the two planes correspond.

The equipotential lines, $G=c, c>0$, take the following forms: (1) the locus $G=c$ consists of a finite number of simple analytic closed curves, mutually exterior, bounding an infinite region $T$ of points $G>c$; or (2) the locus $G=c$ consists of a finite number of mutually exterior closed curves, at least one of which has a multiple point of order $m \geqq 2$, bounding an infinite region $T$ of points $G>c$.

Consider the set of points $T: G>c$, in which we count the point at infinity. Because $G$ is continuous in $K$ except at infinity, the boundary points of $T$ all belong to the equipotential $G=c$. Conversely, all points of $G=c$ are boundary points of $T$. If not, then in a neighborhood of a point $P$ of $G=c$ which is not a boundary point of $T$, we have only points $G \leqq c$. Since $G$ is harmonic in this neighborhood, by Gauss' mean-value theorem $G$ equals $c$ on the circumference of a sufficiently small circle about $P$, and we have a contradiction.

The set $T$ is a region, that is, every point of the set $G>c$ is an interior point of the set, and any two points of the set can be connected by a Jordan arc all of whose points belong to the set.. Otherwise, a region $T_{1}$ belonging to the set $T$ exists not including the point at infinity and having $G=c$ on its entire boundary. Since $G$ is harmonic in $T_{1}, G$ is identically equal to $c$ in $T_{1}$, which leads to a contradiction.

The locus $G=c, c>0$, consists of analytic arcs which fall into a set of closed curves; otherwise the continuity hypothesis is contradicted.*

The locus $G=c, c>0$, consists of a finite number of curves. If $M$ is bounded by a finite number of mutually exclusive closed point sets, the statement follows at once from the facts that any curve on which $G=c>0$ contains in its interior points of $M$ and no two loci $G=0$ and $G=c>0$ have a common point. If $M$ is bounded by an infinite number of mutually exclusive point sets, assume the curves $G=c: C^{(1)}, C^{(2)}, \cdots$ to be infinite in number and consider a point $P_{1}$ on $C^{(1)}, P_{2}$ on $C^{(2)}, \ldots$. Since $G=c$ is a closed limited point set, these points must have a limit point $P$ on $G=c$. If $P$ is not a point at which the gradient of $G$ vanishes, the curve $G=c$ through $P$ is a single

* See for instance Kellogg, Foundations of Potential Theory, Berlin, 1929, pp. 273-277. 
analytic piece, as the theorem on implicit functions shows.* If $P$ is a point at which the gradient vanishes, it is not a limit point of points at which the gradient vanishes, for such points can occur only on the boundary of $K$, as is evident from consideration of the derivative of the analytic function $f(z)$ of which $G$ is the real part. $\dagger$ If $P$ is a point at which the gradient of $G$ vanishes, the analytic arcs of which $G=c$ consists in the neighborhood of $P$ are finite in number and they pass through the point $P$ with equally spaced tangents $\ddagger$, so $P$ cannot be a limit point of points on $C^{(1)}, C^{(2)}, \cdots$ Hence we have reached a contradiction; and the statement that any locus $G=c$, $c>0$, consists of a finite number of curves is true.

The locus $G=c, c>0$, consists either entirely of mutually exterior simple curves, or of mutually exterior curves some of which may be simple but at least one of which, $C^{\prime}$, has a multiple point of order $m, m \geqq 2$; and $C^{\prime}$ contains in its interior (i.e. the finite regions bounded by $C^{\prime}$ ) at least $m$ mutually exclusive closed sets belonging to $M$. The proof is similar to that already given and is left to the reader.

If the region $K$ is of connectivity greater than unity, there is at least one value of $c$ for which the locus $G=c$ contains a curve with a multiple point.

The number of curves of which the locus $G=c$ is composed increases monotonically (if at all) as $c$ decreases. The locus $G=c$ consists of a finite number of mutually exterior simple curves, except for a countable set of values of $c$.

Out of Lemma I follows, as the reader will easily verify,

LEMma II. Under the hypotheses of Lemma I, the point sets bounding the infinite region $K$ can be approximated by finite sets of mutually exterior analytic curves $G=c$. More explicitly, the equipotential loci $J^{(i)}: G=c_{i}, i=1,2, \cdots$, $c_{1}>c_{2}>c_{3}>\cdots \rightarrow 0$, lie in $K$ and are such that the region interior to $J^{(i+1)}$ is contained in the region interior to $J^{(i)}, J^{(i)}$ and $J^{(i+1)}$ have no common points, and every point in $K$ lies exterior to some $J^{(i)}$. If the $c_{i}$ are suitably chosen, each $J^{(i)}$ consists of a finite number of mutually exterior analytic simple curves.

3. Approximation to several analytic curves by a lemniscate. The locus of a point the product of whose distances from $m$ fixed points is constant is a lemniscate. Thus, if the given points are $a_{1}, a_{2}, \cdots, a_{m}$, the lemniscate is defined by the equation $|P(z)|=c$, where $P(z)=\left(z-a_{1}\right)\left(z-a_{2}\right) \cdots\left(z-a_{m}\right)$. For $m=1$, the lemniscate is a circle; for $m=2$, the lemniscate is a Cassinian oval. We note that $|P(z)|=0$ consists of the points $z=a_{i}, i=1,2, \cdots, m$;

* Osgood, Lehrbuch der Funktionentheorie, vol. I, Leipzig, 1923, p. 675.

$\dagger$ The proof follows that of Kellogg in the case that $K$ is simply connected: loc. cit., pp. 364-365.

$\ddagger$ See for instance Kellogg, loc. cit., p. 275. 
and, in the general case, since $G=\log [|P(z)| /|c|]^{1 / m}$ is Green's function with pole at infinity for the region exterior to $|P(z)|=c \neq 0$, the curves $G=\log \epsilon, \epsilon>1$, or $|P(z)|=c \epsilon^{m}$, for $\epsilon$ sufficiently near unity and $c$ sufficiently near zero, are $m$ simple closed analytic curves, each containing one root $a_{i}, i=1,2, \cdots, m$, of $P(z)=0$, if the $a_{i}$ are all distinct.

The possibility of approximation of analytic curves by lemniscates is the basis of our proof of Theorem I, and Theorem I is the source of all succeeding results in this paper.

LEMMA III. A finite number $k$ of arbitrary mutually exterior closed analytic curves can be approximated by the same lemniscate; that is to say, given a set $C$ consisting of $k$ mutually exterior closed analytic curves $C_{j}, j=1,2, \cdots, k$, and a number $\eta>0$ such that the $\eta$-neighborhoods of $C_{j}$ are distinct, a lemniscate $\Gamma:\left|\left(z-a_{1}\right)\left(z-a_{2}\right) \cdots\left(z-a_{m}\right)\right|=c$ exists which lies exterior to $C$ and interior to these $\eta$-neighborhoods, and contains $C$ in its interior.*

Let $C_{j}^{\prime}, j=1,2, \cdots, k$, be $k$ curves constructed as follows:

(1) The curve $C_{j}^{\prime}$ is contained in the region swept out by a circle of radius $\eta$ whose center describes $C_{j}$.

(2) The curve $C_{j}^{\prime}$ contains in its interior one and only one of the given curves, say $C_{j}$, and lies exterior to $C$.

(3) The curves $C_{j}^{\prime}$ lie exterior to one another.

Let $s(\zeta)$ measure arc length on the curves $C_{j}$ whose lengths are $d_{j}$, $j=1,2, \cdots, k$. For $0 \leqq s(\zeta) \leqq d_{1}, \zeta$ shall lie on $C_{1}$; for $d_{1}<s(\zeta) \leqq d_{1}+d_{2}, \zeta$ shall lie on $C_{2} ; \cdots$; for $\sum_{j=1}^{k-1} d_{j}<s(\zeta) \leqq \sum_{j=1}^{k} d_{j}$, $\zeta$ shall lie on $C_{k}$.

Green's function $G(x, y)$ exists $\dagger$ for the region exterior to $C$ : (1) $G(x, y)$ is harmonic exterior to $C$ except at infinity where $G(x, y)=\log r+G_{0}(x, y)$, and $G_{0}(x, y)$ is harmonic at infinity and has the value $-\mu$ at infinity, and (2) $G(x, y)$ is continuous and vanishes on $C$. We define a function $V(x, y)$ so that $V(x, y)=G(x, y)+\mu$; and we now prove that there exists a continuous positive function

$$
\phi(s)=\frac{1}{2 \pi} \frac{\partial V(x, y)}{\partial n}
$$

( $n$ is the exterior normal for $C$ ) such that

* This lemma was proved by Hilbert in the case of approximation to one analytic curve and applied to approximation of analytic functions by polynomials: Göttinger Nachrichten, 1897, pp. 63-70.

Simultaneous approximation of several distinct curves by lemniscates has also been used by other writers, especially Faber, Szegö, Fekete, and P6lya, in connection with the approximation of functions by polynomials and related topics, but without detailed proof of the results of the present paper. See particularly Faber, Münchner Berichte, 1922, pp. 157-178, and for further references P6lya and Szegö, Crelle's Journal, vol. 165 (1931), pp. 4-49.

† Osgood, loc. cit., pp. 687-703. 


$$
V(x, y)=\int_{c} \phi(s) \log r d s,
$$

where now $r=|z-\zeta|, d s=|d \zeta|$, and $z=x+i y$ is any point of the $z$-plane exterior to $C$.

By a familiar theorem of potential theory, a function $G(x, y)$ which is (1) harmonic in the region $S$ which is bounded by $C$ and a circle $C_{0}$ (with center $P:(x, y)$ exterior to $C)$ containing $C$ in its interior, and (2) continuous together with its partial derivatives of the first order on the boundary of $S$, satisfies the following equation:

$$
G(x, y)=\frac{1}{2 \pi} \int_{c_{0}}\left(\log r \frac{\partial G}{\partial n}-G \frac{\partial \log r}{\partial n}\right) d s
$$

(a)

$$
+\frac{1}{2 \pi} \int_{C}\left(\log r \frac{\partial G}{\partial n}-G \frac{\partial \log r}{\partial n}\right) d s .
$$

Here $r$ denotes distance from $P:(x, y)$ and $n$ denotes interior normal with respect to $S$.

If we use the Green's function $G(x, y)=\log r+G_{1}(x, y)$, we have from (a)

$$
G(x, y)=-\mu+\frac{1}{2 \pi} \int_{c} \log r \frac{\partial G}{\partial n} d s,
$$

for we have

$$
\begin{array}{r}
G(x, y)=\frac{1}{2 \pi} \int_{C_{0}}\left(\log r \frac{\partial G_{1}}{\partial n}-G_{1} \frac{\partial \log r}{\partial n}\right) d s+\frac{1}{2 \pi} \int_{C} \log r \frac{\partial G}{\partial n} d s, \\
\int_{C_{0}} \log r \frac{\partial G_{1}}{\partial n} d s=0, \quad \frac{1}{2 \pi} \int_{C_{0}} G_{1} \frac{\partial \log r}{\partial n} d s=\frac{1}{2 \pi} \int_{C_{0}} G_{0} \frac{\partial \log r}{\partial n} d s=\mu .
\end{array}
$$

Consequently,

and

$$
G(x, y)+\mu=\frac{1}{2 \pi} \int_{C} \log r \frac{\partial(G+\mu)}{\partial n} d s,
$$

$$
V(x, y)=\frac{1}{2 \pi} \int_{c} \log r \frac{\partial V}{\partial n} d s .
$$

The function $\partial V / \partial n$ is continuous on $C$ since $\partial G / \partial n$ is continuous on $C$, and $\partial V / \partial n$ is positive since $V(x, y)$ is harmonic exterior to $C$ except at infinity where it is logarithmically infinite. Hence 


$$
\frac{1}{2 \pi} \frac{\partial V}{\partial n}=\phi(s)
$$

is the function desired.

Since $V(x, y)$ is harmonic exterior to $C$ except at infinity, $V(x, y)$ takes on all the $C_{j}^{\prime}$ a minimum value $\mu_{1}>\mu$. We now choose $\epsilon>0$ such that $\epsilon<\left(\mu_{1}-\mu\right) / 2$. Since $V(x, y)-\mu$ is Green's function for the region exterior to $C$ we may apply Lemma I. If $V=\mu+\epsilon$ is a locus consisting of curves not all of which are simple, some curve of the locus must intersect a $C_{j}^{\prime}$. Since $\mu+\epsilon<\mu_{1}$, and $\mu_{1}$ is the minimum value of $V$ on $C_{j}^{\prime}$, the curve cannot cut $C_{j}^{\prime}$. Hence $V=\mu+\epsilon$ consists of a simple closed curve $\gamma_{1}$ in the ring $C_{1} C_{1}^{\prime}$, a simple closed curve $\gamma_{2}$ in the ring $C_{2} C_{2}^{\prime}, \cdots$, a simple closed curve $\gamma_{k}$ in the ring $C_{k} C_{k}^{\prime}$.

By similar reasoning, $V=\mu_{1}-\epsilon$ consists of a simple closed curve $\gamma_{1}^{\prime}$ in the ring $C_{1} C_{1}^{\prime}$, a simple closed curve $\gamma_{2}^{\prime}$ in the ring $C_{2} C_{2}^{\prime}, \cdots$, a simple closed curve $\gamma_{k}^{\prime}$ in the ring $C_{k} C_{k}^{\prime}$.

We denote by $\gamma_{j} \gamma_{j}^{\prime}$ the ring region bounded by $\gamma_{j}$ and $\gamma_{j}^{\prime}$. We let

$$
u_{0}=\int_{0}^{d_{1}+d_{2}+\cdots+d_{k}} \phi(s) d s ;
$$

and we make the change of variable

$$
u(\zeta)=\int_{0}^{s(\zeta)} \phi(s) d s
$$

so that $u$ increases from 0 to $u_{0}$ as $s$ increases from 0 to $\sum_{j=1}^{k} d_{j}$. Then

$$
V(x, y)=\int_{0}^{u_{0}} \log r d u,
$$

and

$$
V(x, y)=\lim _{n \rightarrow \infty}\left(u_{0} / n\right)\left(\log r_{1}+\log r_{2}+\cdots+\log r_{n}\right)
$$

where $r_{1}, r_{2}, \cdots, r_{n}$ are distances from $z$ to $n$ points of $C_{j}$ corresponding to equidistant values $u_{1}=u_{0} / n, u_{2}=2 u_{0} / n, \cdots, u_{n}=u_{0}$ of $u$. For simplicity, the dependence of $r_{i}$ on $n$ is not indicated in the notation.

For $z$ interior to $\gamma_{j} \gamma_{j}^{\prime}$, convergence of the sequence of functions $u_{0} \log r_{1}$, $\left(u_{0} / 2\right)\left(\log r_{1} r_{2}\right), \cdots,\left(u_{0} / N\right)\left(\log r_{1} r_{2} \cdots r_{n}\right), \cdots$, to $V(x, y)$ is uniform.* For $n$ sufficiently large, $n \geqq N$, and $z$ interior to $\gamma_{j} \gamma_{j}^{\prime}$, we have

$$
-\epsilon^{\prime}<V(x, y)-\left(u_{0} / n\right)\left(\log r_{1} r_{2} \cdots r_{n}\right)<\epsilon^{\prime} .
$$

* The detailed proof of uniformity offers no difficulty. See for instance Walsh, Bulletin of the American Mathematical Society, vol. 35 (1929), pp. 499-544; Lemma, p. 538. 
If we denote by $\Gamma$ the locus exterior to $C:\left(u_{0} / N\right)\left(\log r_{1} r_{2} \cdots r_{N}\right)=\lambda$, where $\mu+\epsilon<\lambda<\mu_{1}-\epsilon$, and choose $\epsilon^{\prime}$ sufficiently small, we have, on $\Gamma$, $\mu+\epsilon<\lambda-\epsilon^{\prime}<V(x, y)<\lambda+\epsilon^{\prime}<\mu_{1}-\epsilon$. Every Jordan arc joining a point of $\gamma_{j}$ to a point of $\gamma_{j}^{\prime}$ must cut $\Gamma$. The locus $\Gamma$ has the following properties:

(1) $\Gamma$ consists of a curve $\Gamma^{(1)}$ enclosing $\gamma_{1}$, a curve $\Gamma^{(2)}$ enclosing $\gamma_{2}, \cdots$, and a curve $\Gamma^{(k)}$ enclosing $\gamma_{k}$. Otherwise there would exist a region in some $\gamma_{j} \gamma_{j}^{\prime}$ in which the harmonic function $\left(u_{0} / N\right)\left(\log r_{1} r_{2} \cdots r_{N}\right)$ constant on $\Gamma$ would be identically constant, which is impossible.

(2) $\Gamma$ lies in the rings $\gamma_{j} \gamma_{j}^{\prime}$, since $\lambda$ is such that $\mu+\epsilon<\lambda-\epsilon^{\prime}<V(x, y)<\lambda$ $+\epsilon^{\prime}<\mu_{1}-\epsilon$.

(3) $\Gamma$ is a lemniscate, for the equation

$$
r_{1} r_{2} \cdots r_{N}=e^{N \lambda / u_{0}}
$$

is of the form $|P(z)|=c>0$.

The proof of Lemma III is now complete.

If we choose $\eta$ successively $1,1 / 2, \cdots, 1 / n, \cdots$, we have lemniscates $\Gamma_{1}, \Gamma_{2}, \cdots, \Gamma_{n}, \cdots$, exterior to $C$. From this set can be extracted a subset such that (1) each $\Gamma_{i_{0}+1}$ is interior to $\Gamma_{i_{0}}$, (2) $\Gamma_{i_{0}}$ and $\Gamma_{i_{0}+1}$ have no common point, and (3) every point exterior to $C$ lies exterior to some $\Gamma_{i_{0}}$.

4. Lemmas involving the mapping function $\omega(z)$. We prove the following lemmas:

LEMMA IV. Under the hypotheses of Lemma I, a multiple point of order $m$ of the curves $G=c, c>0$, occurs at $(x, y)=\left(x^{\prime}, y^{\prime}\right)$ if and only if $\omega^{\prime}\left(z^{\prime}\right)=0$, $\omega^{\prime \prime}\left(z^{\prime}\right)=0, \cdots, \omega^{(m-1)}\left(z^{\prime}\right)=0, \omega^{(m)}\left(z^{\prime}\right) \neq 0, z^{\prime}=\left(x^{\prime}, y^{\prime}\right), m \geqq 2$, simultaneously, that is, when and only when $z=z^{\prime}$ is a branch point of the inverse of the mapping function $\omega(z)=e^{G+i B}$.

The proof of this lemma is essentially included in Lemma $I$.

Lemma V. Let $M, K, \omega(z)$ be defined as in Theorem I. Let $C_{R}$ denote* the transform in the z-plane of $|w|=R, R>1$, under the mapping function $\omega(z)$. Let $\rho$ be arbitrary, $1<\rho<R$. Then there exists a lemniscate (of Lemma III) $\Gamma:\left|\left(z-a_{1}\right) \cdots\left(z-a_{m}\right)\right|=c$ such that $\Gamma$ contains $M$ in its interior and such that $\Gamma_{R / \rho}:\left|\left(z-a_{1}\right) \cdots\left(z-a_{m}\right)\right|=c R^{m} / \rho^{m}$ lies interior to $C_{R}$. Thus for $z$ on and within $\Gamma$ (hence on $M$ ) and for $t$ on or exterior to $\Gamma_{R / \rho}$, we have

$$
\left|\frac{\left(z-a_{1}\right) \cdots\left(z-a_{m}\right)}{\left(t-a_{1}\right) \cdots\left(t-a_{m}\right)}\right| \leqq \frac{\rho^{m}}{R^{m}} .
$$

* A symbol of the form $C_{R}$ denotes henceforth the transform in the z-plane of $|w|=R, R>1$, under the mapping function $\omega(z)$. 
Let $\Gamma$ be a lemniscate contained in $K$ and lying interior to $C_{\rho}$. Then the locus $\Gamma_{R / \rho}$ lies interior to $\left[C_{\rho}\right]_{R / \rho}=C_{R}$, as follows from the study of the Green's functions for the exterior of $C_{\rho}$ and the exterior of $\Gamma$. If these Green's functions are denoted by $G_{1}$ and $G_{2}$ respectively, their difference $G_{1}-G_{2}$ is negative on $C_{\rho}$, hence harmonic and negative exterior to $C_{\rho}$ even at infinity. Then on $\Gamma_{R / \rho}$ we have $G_{1}-\log R / \rho<0$, so on $\Gamma_{R / \rho}$ we have $G_{1}<\log R / \rho$; the curve $C_{R}: G_{1}=\log R / \rho$ lies exterior to $\Gamma_{R / \rho}$.

Lemma VI. Let $M, K, \omega(z)$ be defined as in Theorem I. If $Q(z)$ is a polynomial of degree $n$ such that $|Q(z)| \leqq L, z$ on $M$, then

$$
|Q(z)| \leqq L R_{0}^{n}, z \text { on and within } C_{R_{0}}, R_{0}>1 \text {. }
$$

The special case in which $M$ is a line segment is due to S. Bernstein,* and the method used in proving Lemma VI is a generalization of the method of M. Riesz $\dagger$ for this special case. This lemma was proved by Walsh $\ddagger$ in case $K$ is simply connected and the possibility of its extension to the more general case was indicated by him. See also Faber (loc. cit.), who proves Lemma VI for a set $M$ bounded by a finite number of Jordan curves.

5. Approximation to an analytic function. We proceed now to the proof of Theorem I.

We first prove (1). Since $f(z)$ is analytic on and within $C_{R}$, the function $f(z)$ is also analytic on and within some $C_{R^{\prime}}, R^{\prime}>R$. Choose the present $R^{\prime}$ as the $R$ of Lemma V and the present ratio $R^{\prime} / R$ as the quantity $\rho$ of Lemma V. Then by Lemma $\mathrm{V}$ there exists a lemniscate $\Gamma:\left|\left(z-a_{1}\right) \cdots\left(z-a_{m}\right)\right|=c$ containing $M$ in its interior, while $\Gamma_{R}:\left|\left(\dot{z}-a_{1}\right) \cdots\left(z-a_{m}\right)\right|=c R^{m}$ is interior to $C_{R^{\prime}}$; for $z$ on and within $\Gamma$ (in particular on $M$ ) and for $t$ on or exterior to $\Gamma_{R}$ (in particular on $C_{R^{\prime}}$ ), we have

$$
\left|\frac{\left(z-a_{1}\right) \cdots\left(z-a_{m}\right)}{\left(t-a_{1}\right) \cdots\left(t-a_{m}\right)}\right| \leqq \frac{1}{R^{m}}
$$

A unique polynomial $P_{m p-1}(z)$ of degree $m p-1, p=1,2, \cdots$, exists with the properties

$$
P_{m p-1}^{(i)}\left(a_{j}\right)=f^{(i)}\left(a_{j}\right) \quad(i=0,1,2, \cdots, p-1 ; j=1,2, \cdots, m) . \S
$$

* Mémoires de l'Académie Royale de Belgique, Classe des Sciences, (2), vol. 4 (1912), pp. 36-94.

$\dagger$ Acta Mathematica, vol. 40 (1916), pp. 337-347.

$\ddagger$ Münchner Berichte, loc. cit., p. 225.

$\S$ Hilbert (loc. cit.) has exhibited such polynomials in the case of approximation in a simply connected region. See also Jacobi, Crelle's Journal, vol. 53 (1856-57), pp. 103-126; and Montel, Leçons sur les Séries de Polynomes, Paris, 1910, pp. 47-49, 95-97. 
Two distinct polynomials $P_{m p-1}(z)$ of degree $m p-1$ surely cannot satisfy these conditions, for their difference would have at least $m p$ roots. We actually exhibit the polynomial $P_{m p-1}(z)$ (necessarily unique):

$P_{m p-1}(z)=f(z)-\frac{1}{2 \pi i} \int_{C_{R^{\prime}}} \frac{f(t)}{t-z}\left[\frac{\left(z-a_{1}\right) \cdots\left(z-a_{m}\right)}{\left(t-a_{1}\right) \cdots\left(t-a_{m}\right)}\right]^{p} d t, \quad z$ interior to $C_{R^{\prime}} \ldots$

Indeed, it is clear by inspection that $P_{m p-1}(z)$ thus defined satisfies the conditions on interpolation to $f(z)$, since this equation is valid for $z=a_{j}$. Moreover, if $f(z)$ is expressed by Cauchy's integral (which may be taken over the whole of $C_{R^{\prime}}$ even if $C_{R^{\prime}}$ consists of several curves):

$$
f(z)=\frac{1}{2 \pi i} \int_{C_{R}, t-z} \frac{f(t)}{t-z} d t, z \text { interior to } C_{R^{\prime}},
$$

substitution in the previous equation leads to an integrand which has no singularity in $z$ and which is a polynomial in $z$ of degree $m p-1$, so the function $P_{m p-1}(z)$ is seen to be a polynomial of degree $m p-1$.

For $z$ on $M$, we have

$$
\left|f(z)-P_{m p-1}(z)\right| \leqq \frac{1}{2 \pi} \int_{C_{R^{\prime}}} \frac{|f(t)|}{|t-z|}\left|\frac{\left(z-a_{1}\right) \cdots\left(z-a_{m}\right)}{\left(t-a_{1}\right) \cdots\left(t-a_{m}\right)}\right|^{p}|d t| .
$$

Since $f(z)$ is analytic on and within $C_{R^{\prime}}$, there follow the inequalities $|f(t)|$ $\leqq N^{\prime \prime} ;\left|\left(z-a_{1}\right) \cdots\left(z-a_{m}\right)\right| /\left|\left(t-a_{1}\right) \cdots\left(t-a_{m}\right)\right| \leqq 1 / R^{m}$, and $1 /|t-z|$ $\leqq 1 / \delta, z$ on $M, t$ on $C_{R^{\prime}}$. Set

$$
\int_{C_{R^{\prime}}}|d t|=L
$$

we have

$$
\begin{aligned}
\left|f(z)-P_{m p-1}(z)\right| & \leqq \frac{1}{2 \pi} \cdot \frac{1}{R^{m p}} \cdot \frac{N^{\prime \prime} L}{\delta} \\
& \leqq \frac{N^{\prime}}{R^{m p}}, z \text { on } M
\end{aligned}
$$

where $N^{\prime}$ is independent of $p$ and $z$.

The polynomial $P_{n}(z)$ of degree $n, n=1,2, \cdots$, already defined when $n$ is of the form $m p-1$, is now defined for arbitrary $n$ by the equation

$$
P_{n}(z)=P_{m p-1}(z), \quad m p-1 \leqq n<m(p+1)-1 .
$$

Then we have the inequality 


$$
\left|f(z)-P_{n}(z)\right| \leqq \frac{N^{\prime}}{R^{n-m+2}} \leqq \frac{N}{R^{n}}, z \text { on } M,
$$

where $N=N^{\prime} R^{m-2}$, and where $N$ is independent of $n$ and $z$. The proof of the first part of the theorem is complete.

The proof of the second part of the theorem is the analog of the corresponding proof given by Walsh in the case of a closed limited point set whose complement is simply connected, ${ }^{*}$ and is a direct application of Lemma VI.

The following theorem is simpler but less explicit than Theorem I:

THEOREM. Let $M$ be an arbitrary closed limited point set whose complement $K$ is connected and possesses a Green's function with pole at infinity. A necessary and sufficient condition that $f(z)$ be analytic on $M$ is that there exist polynomials $P_{n}(z)$ of respective degrees $n$ such that the inequality

$$
\left|f(z)-P_{n}(z)\right| \leqq \frac{N}{R^{n}}, R>1,
$$

$N$ not dependent on $n$ or $z$, is valid for every $z$ on $M$.

The function $f(z)$ of Theorem I is not necessarily a monogenic analytic function; in other words, if we consider the functions defined on various separated pieces of $M$, the hypotheses of the theorem may well be satisfied where $f(z)$ is not a monogenic analytic function.

Theorem II shows the best degree of approximation (measured like the convergence of a geometric series) possible for a sequence of polynomials $\left\{P_{n}(z)\right\}$ :

Theorem II. Let $M, K, \omega(z)$ be defined as in Theorem I, and let $f(z)$ be analytic on $M$. Let $R$ be the largest number for which the following is true: (1) a function $F(z)$ is analytic and single-valued interior to $C_{R}$, (2) $F(z) \equiv f(z)$ on $M$. Then there exists a sequence of polynomials $\left\{P_{n}(z)\right\}$ of respective degrees $n$, $n=1,2, \cdots$, such that

$$
\left|f(z)-P_{n}(z)\right| \leqq \frac{N}{R_{0}^{n}}, \text { on } M, R_{0} \text { arbitrary }<R,
$$

$N$ dependent on $R_{0}$ but not on $n$ or $z ;$ but for no sequence of polynomials $\left\{P_{n}(z)\right\}$ do we have

$$
\left|f(z)-P_{n}(z)\right| \leqq \frac{N}{R_{1}^{n}}, z \text { on } M, R_{1}>R,
$$

$N$ dependent on $R_{1}$ but not on $n$ or $z$.

* Münchner Berichte, loc. cit., p. 226. 
The number $R$ defined by (1) and (2), finite or infinite, exists; the formal proof is left to the reader.

The curve $C_{R}$ is characterized by the fact that the function $f(z)$. (when suitably extended analytically from $M$ along paths interior to $C_{R}$ ) is analytic and single-valued interior to $C_{R}$, but is not analytic or is not single-valued or fails in both particulars interior to every $C_{R^{\prime}}, R^{\prime}>R$, when extended from $M$ along paths interior to $C_{R^{\prime}}$. Thus, (a) at some point $P$ of $C_{R}$ the function $f(z)$ has a singularity for analytic extensions from $M$ along paths interior to $C_{R}$ terminating in $P$; or (b) the curve $C_{R}$ has at least one multiple point $Q$, and there is disagreement at $Q$ among the various analytic extensions of $f(z)$ from the various parts of $M$ to $Q$ along paths belonging to the several regions interior to and bounded by $C_{R}$; or (c) both (a) and (b) occur.

As an illustration of (b) let the point set $M$ be the closed interior of the lemniscate $\left|z^{2}-1\right|=c, c<1$, and let $f(z)=1$ in the oval to the right of the origin, and $f(z)=-1$ in the oval to the left of the origin. Then $C_{R}$ is the lemniscate $\left|z^{2}-1\right|=1$. As an illustration of (c) let $f(z)=1 /\left(z-2^{1 / 2}\right)$ and $1 /\left(z+2^{1 / 2}\right)$ in the right and left ovals of the point set $M$ above. Then $C_{R}$ is again the lemniscate with double point $\left|z^{2}-1\right|=1$.

The first statement of Theorem II has been proved in Theorem I, although the polynomials there exhibited depend on $R_{0}$; this restriction does not appear for the polynomials of Theorem III below. We shall prove the second statement.

Assume that polynomials $P_{n}(z)$ of degree $n, n=1,2, \cdots$, exist such that

$$
\left|f(z)-P_{n}(z)\right| \leqq \frac{N}{R_{1}^{n}}, z \text { on } M, R_{1}>R,
$$

$N$ independent of $n$ and $z$. By Theorem $\mathrm{I}$, the sequence $\left\{P_{n}(z)\right\}$ converges to an analytic function $F(z)$ within $C_{R_{1}}$. Then $F(z)$ is analytic interior to $C_{R_{1}}$ and $F(z) \equiv f(z)$ on $M$, where $R_{1}$ is greater than $R$, contrary to hypothesis.

6. The Tchebycheff polynomial. The Tchebycheff polynomial of degree $n$ for approximation to $f(z)$ on $M$ is the polynomial $\Pi_{n}(z)$ of degree $n$ such that

$$
\max \left|f(z)-\Pi_{n}(z)\right|, z \text { on } M,
$$

is not greater than the corresponding expression for any other polynomial of degree $n$. The Tchebycheff polynomial exists and is unique, ${ }^{*}$ under the hypotheses of Theorem I.

Theorem III states the exact region of uniform convergence of sequences of polynomials of best approximation in the sense of Tchebycheff. The first

* Tonelli, Annali di Matematica, vol. 15 (1908), pp. 47-119. 
part of this theorem was proved by Faber* for a point set $M$ bounded by an analytic Jordan curve and the entire theorem was proved by Walsh $\dagger$ in the case of a closed limited point set whose complement is simply connected.

THEOREM III. Under the hypotheses of Theorem II, the sequence of polynomials $\left\{\Pi_{n}(z)\right\}$ of respective degrees $n, n=1,2, \cdots$, of best approximation in the sense of $T$ chebycheff to $f(z)$ on $M$ converges interior to $C_{R}$, uniformly on any closed point set interior to $C_{R}$, and converges uniformly in no region containing a point of $C_{R}$ in its interior.

The proof of this theorem is the analog of that given by Walsh in the case of a closed limited point set whose complement is simply connected, and is omitted.

The proof of Theorem III holds for the following theorem:

Any other sequence of polynomials which converges on $M$ like the Tchebycheff polynomials, or, in other words, such that the inequality $\left|f(z)-P_{n}(z)\right| \leqq N / R_{0}^{n}$ is satisfied for $z$ on $M$ and for $R_{0}$ arbitrary less than $R$, where $N$ depends on $R_{0}$ but not on $z$, converges as in Theorem III.

The following theorem was proved by Walsh $\ddagger$ in the special case of a point set whose complement is simply connected:

Under the hypotheses of Theorem III, neither the sequence of polynomials $\left\{\Pi_{n}(z)\right\}$ of best approximation to $f(z)$ on $M$ nor any other sequence of polynomials which converges like the sequence of polynomials of best approximation converges like a geometric series in any region or on any Jordan arc exterior to $C_{R}$.

The proof follows the method of proof of the last part of Theorem III.

In particular, the discussion holds for simultaneous approximation to real analytic functions on a finite number of intervals on the axis of reals.

7. Other measures of approximation. There are other measures of approximation such as (1) approximation by the Tchebycheff method with a norm function, (2) approximation on $M$ as measured in the sense of least $p$ th powers $(p>0)$ by line integrals in the case that $M$ (closed, limited) is bounded by a finite number of rectifiable Jordan curves, (3) approximation on $M$ as measured in the sense of least $p$ th powers $(p>0)$ by surface integrals where $M$ (closed, limited, consisting of a finite number of regions) is an open set plus its boundary points.

In each of these cases the polynomial of best approximation exists, and is

* Crelle's Journal, vol. 150 (1920), pp. 79-106.

$\dagger$ (1), p. 795; (2), pp. 381-384.

$\ddagger(2)$, p. 385 . 
unique if $p>1 .^{*}$ In each case, as we shall proceed to indicate, under suitable restrictions on $M$, the sequence of polynomials of best approximation to $f(z)$ on $M$ converges satisfying the inequality $\left|f(z)-P_{n}(z)\right| \leqq N / R_{0}^{n}, R_{0}<R, z$ on $M$, and hence converges interior to $C_{R}$, uniformly on any closed set interior to $C_{R}$, but converges uniformly in no region containing a point of $C_{R}$ in its interior.

The proofs in each of these cases are analogous to proofs already given by Walsh. $\dagger$ In cases (2) and (3), an inequality of form $\left|f(z)-P_{n}(z)\right| \leqq N / R_{0}^{n}$, $R_{0}<R$, is first proved not for $z$ on $M$ but for $z$ on a suitably chosen closed set $M^{\prime}$ interior to $M$. The conclusion follows from the fact that when $M^{\prime}$ approaches $M$, then $C_{R}{ }^{\prime}$ (defined for $M^{\prime}$ as $C_{R}$ is defined for $M$ ) approaches $C_{R}$; this latter fact is a consequence of the fundamental results of Lebesgue $\ddagger$ on harmonic functions and variable domains.

A Tchebycheff polynomial for approximation to $f(z)$ on $M$ with the norm function $p(z)$, where $p(z)$ is given continuous and different from zero on $M$, is the unique polynomial $\Pi_{n}^{\prime}(z)$ of degree $n$ such that

$$
\max \left[|p(z)|\left|f(z)-\Pi_{n}^{\prime}(z)\right|\right], z \text { on } M,
$$

is not greater than the corresponding expression for any other polynomial of degree $n$.

THEOREM IV. Under the hypotheses of Theorem II, the sequence of Tchebycheff polynomials $\left\{\Pi_{n}^{\prime}(z)\right\}$ for approximation to $f(z)$ on $M$ with an arbitrary positive continuous norm function $p(z)$ converges interior to $C_{R}$, uniformly on an arbitrary closed point set interior to $C_{R}$, and converges uniformly in no region containing a point of $C_{R}$ in its interior.

A polynomial of best approximation in the sense of least weighted $p$ th powers as measured on $\sum \Gamma_{j}$, where $\Gamma_{j}, j=1,2, \cdots, k$, are $k$ rectifiable Jordan curves bounding the point set $M$ (satisfying the hypotheses of Theorem I), is a polynomial $\Pi_{n}(z)$ of degree $n$ such that

$$
\sum_{j=1}^{k} \int_{\mathrm{r}_{j}}\left|f(z)-\Pi_{n}(z)\right|^{p} n(z) d z,
$$

where $p>0$ and $n(z)$ is arbitrary, continuous, positive, is not greater than the corresponding expression formed for any other polynomial of degree $n$.

THEOREM V. Let the closed limited point set $M$ whose complement is connected be bounded by a finite number $k$ of non-intersecting rectifiable Jordan

\footnotetext{
* See for instance Walsh, these Transactions, vol. 33 (1931), pp. 668-689; p. 681.

$\dagger(1) ;(2)$.

$\ddagger$ Palermo Rendiconti, vol. 24 (1907), pp. 371-402.
} 
curves $\Gamma_{j}$. Under the hypotheses of Theorem II, the sequence of polynomials $\left\{\Pi_{n}(z)\right\}$ of best approximation to $f(z)$ on $M$ in the sense of least weighted pth powers $(p>0)$ as measured on $\Gamma_{j}$ converges throughout the interior of $C_{R}$, uniformly on any closed point set interior to $C_{R}$, and converges uniformly in no region containing a point of $C_{R}$ in its interior.

The case $p=2$ is of especial interest. Here the polynomial $\Pi_{n}(z)$ of best approximation to an arbitrary function $f(z)$ is of the form

$$
\Pi_{n}(z)=a_{0} P_{0}(z)+a_{1} P_{1}(z)+\cdots+a_{n} P_{n}(z),
$$

where the $P_{i}(z), i=1,2, \cdots, n$, depend on the $\Gamma_{j}$ but not on $f(z)$, and the coefficients $a_{i}(i \leqq n)$ are independent of $n$. The set of polynomials $P_{i}(z)$ is said to belong to the point set $M$.

The method of approximation used in Theorem $\mathrm{V}$ for $p=2, n(z) \equiv 1$, was discussed and the corresponding special case of Theorem $V$ was proved (under an additional restriction) by Szegö* and Smirnoff $\dagger$ for the case of a point set whose complement is simply connected.

A polynomial of best approximation in the sense of least weighted $p$ th powers as measured by integration over the areas $\bar{R}_{j}$, where $\bar{R}_{j}, j=1,2, \ldots$, $k$, are arbitrary closed regions, is a polynomial $\Pi_{n}(z)$ of degree $n, n=1,2, \cdots$, such that

$$
\sum_{j=1}^{k} \iint_{\bar{R}_{j}}\left|f(z)-\Pi_{n}(z)\right|^{p} n(z) d S,
$$

where $p>0, n(z)$ is continuous and positive in $\bar{R}_{j}$, is not greater than the corresponding expression $\ddagger$ formed for any other polynomial of degree $n$.

TheOREM VI. Let $\bar{R}_{j}, j=1,2, \cdots, k$, be arbitrary closed limited regions no two of which have a common point. Let $K$ denote the region consisting of all points which can be connected with the point at infinity by Jordan arcs which do not contain points of the $\bar{R}_{j}$. Let $G(x, y)$ be Green's function with pole at infinity for $K$. Under the hypotheses of Theorem II, the sequence $\left\{\Pi_{n}(z)\right\}$ of polynomials of best approximation to $f(z)$ in the sense of least weighted pth powers, $p>0$, over the areas $\bar{R}_{j}, j=1,2, \cdots, k$, converges interior to $C_{R}$, uniformly on any closed point set interior to $C_{R}$, and converges uniformly in no region containing a point of $C_{R}$ in its interior.

It will be noticed that the regions $\bar{R}_{j}$ are not necessarily Jordan regions, and in fact any region $\bar{R}_{j}$ may be multiply connected and even if simply con-

* Mathematische Zeitschrift, vol. 9 (1921), pp. 218-270.

$\dagger$ Journal de la Société Physico-Mathématique de Léningrade, vol. 2 (1928), pp. 155-178.

$\ddagger$ If any of the boundaries of $\bar{R}_{i}, j=1,2, \cdots, k$, have area, either upper or lower integral may be used here. 
nected may separate various regions $B$ from $K$. The hypothesis of Theorem VI includes the analyticity of $f(z)$ in all such regions $B$.

The case $p=2$ is again of especial interest. The polynomial $\Pi_{n}(z)$ of best approximation to an arbitrary function $f(z)$ is of the form

$$
\Pi_{n}(z)=a_{0} P_{0}(z)+a_{1} P_{1}(z)+\cdots+a_{n} P_{n}(z),
$$

where the $P_{i}(z), i=1,2, \cdots, n$, depend on the $\bar{R}_{j}$ but not on $f(z)$, and the coefficients $a_{i}(i \leqq n)$ are independent of $n$. The set of polynomials $P_{i}(z)$ is said to belong to the point set $M$.

The method of approximation used in Theorem VI was considered by Bergmann,* Bochner, $\dagger$ and Carleman $\ddagger$ in the case of a single Jordan region, $p=2, n(z) \equiv 1$, although without proof of our results on degree of convergence and overconvergence.

As a complement to Theorems IV-VI we add

THEOREM VII. If $M$ consists of a finite number of mutually exclusive closed Jordan regions and if the function $f(z)$ is analytic in the interior points of $M$, continuous in the corresponding closed regions, then (1) the sequence of polynomials of best approximation to $f(z)$ on $M$ in the sense of Tchebycheff with a positive continuous norm function converges to $f(z)$ uniformly on $M$; (2) if the Jordan curves bounding $M$ are rectifiable, the sequence of polynomials of best approximation to $f(z)$ on $M$ in the sense of least pth powers $(p>0)$ as measured by a line integral with a positive continuous norm function converges to $f(z)$ at every interior point of $M$, uniformly on any closed set interior to $M$; (3) the sequence of polynomials of best approximation to $f(z)$ on $M$ in the sense of least pth powers $(p>0)$ as measured by a surface integral with a positive continuous norm function converges to $f(z)$ at every interior point of $M$, uniformly on any closed set interior to $M$.

In case (3) it is indeed sufficient (see Carleman, loc. cit.) for this conclusion if $f(z)$ is analytic interior to $M$ and if $\iint_{M}|f(z)|^{2} d S$ exists; the restrictions in case (2) can similarly be somewhat lightened (see Smirnoff, loc. cit.).

HARVARD UNIVERSTTY,

CAMBridge, Mass.

Wellesley College,

WeLLesley, Mass.

* Mathematische Annalen, vol. 86 (1922), pp. 238-271.

$\dagger$ Mathematische Zeitschrift, vol. 14 (1922), pp. 180-207.

‡ Arkiv för Matematik, Astronomi, och Fysik, vol. 17 (1922-23). 